\title{
Preparing Greenberger-Horne-Zeilinger Entangled Photon Fock States of Three Cavities Coupled by a Superconducting Flux Qutrit
}

\author{
Zhen-Fei Zheng ${ }^{2}$, Qi-Ping $\mathrm{Su}^{1}$, and Chui-Ping Yang ${ }^{1,3}$ 米 \\ ${ }^{1}$ Department of Physics, Hangzhou Normal University, Hangzhou, Zhejiang 310036, China \\ ${ }^{2}$ Jing Hengyi Honors College, Hangzhou Normal University, Hangzhou, Zhejiang 310036, China and \\ ${ }^{3}$ State Key Laboratory of Precision Spectroscopy, Department of Physics, \\ East China Normal University, Shanghai 200062, China
}

(Dated: September 28, 2018)

\begin{abstract}
We propose a way to prepare Greenberger-Horne-Zeilinger (GHZ) entangled photon Fock states of three cavities, by using a superconducting flux qutrit coupled to the cavities. This proposal does not require the use of classical microwave pulses and measurement during the entire operation. Thus, the operation is greatly simplified and the circuit engineering complexity and cost is much reduced. The proposal is quite general and can be applied to generate three-cavity GHZ entangled photon Fock states when the three cavities are coupled by a different three-level physical system such as a superconducting charge qutrit, a transmon qutrit, or a quantum dot.
\end{abstract}

PACS numbers: 03.67.-a, 42.50.Pq, 85.25.-j

\section{INTRODUCTION}

Entanglement is one of the important properties, through which a quantum physical system can be identified to be distinguished from a classical physical system. On the other hand, entanglement is one of the cornerstones in building up a quantum machine. It plays an important role in both quantum information processing (QIP) and quantum communication (e.g., quantum teleportation, quantum secret sharing, quantum key distribution and so on). Over the past decade, experimental preparation of entanglement has been reported with various of physical systems, such as eight photons via linear optical devices [1], fourteen trapped ions [2], three spins [3], two atoms in microwave cavity QED [4], two atoms plus one cavity mode [5], and two excitions in a single quantum dot [6]. In addition, many schemes have been proposed for generating entangled states of superconducting qubits based on cavity QED [7] or via capacitive couplings [8]. Moreover, various two-qubit or three-qubit entangled states have been experimentally demonstrated with superconducting qubits coupled to a single cavity [9-13].

Superconducting devices [14-16] play significant roles in scalable quantum computing. The physical system composed of cavities and superconducting qubits is one of the most promising candidates for QIP $[14,15]$. Superconducting qubits, such as flux, charge and phase qubits, have long decoherence time $[17,18]$ and experiments have realized quantum operations in single and multiple superconducting qubits with states read out [19-23]. A cavity (such as coplanar waveguide, microstrip resonator and lumped-circuit resonator and so on) can act as a quantum bus, which can mediate long-distance and fast interaction between distant superconducting qubits [24-29]. In addition, superconducting qubits and microwave resonators can be fabricated with modern technology. Furthermore, the strong coupling limit between the superconducting qubits and the cavity field was predicated earlier $[29,30]$ and has been experimentally demonstrated [31,32].

So far, many theoretical proposals have been presented for the preparation of Fock states, coherent states, squeezed states, the Schördinger Cat state, and an arbitrary superposition of Fock states of a single superconducting cavity [3336]. Recently, there is much interest in generation of entangled states of qubits or photons in multiple cavities because of their importance in realizing large-scale QIP within cavity QED. Several theoretical proposals for generation of entangled photon Fock states of two resonators by a superconducting coupler qubit $[37,38]$ have been presented. A theoretical proposal for the manipulation and generation of nonclassical microwave field states as well as the creation of controlled multipartite entanglement with two resonators coupled by a superconducting qubit has been also presented [39]. Based on the resonant interaction with a tunable superconducting phase qutrit coupled to two resonators, an efficient proposal for generating NOON states has been proposed recently [40]. Moreover, by using a phase qutrit coupled to two resonators, a recent experimental demonstration of an entangled NOON state of photons in two

*E-mail: yangcp@hznu.edu.cn 


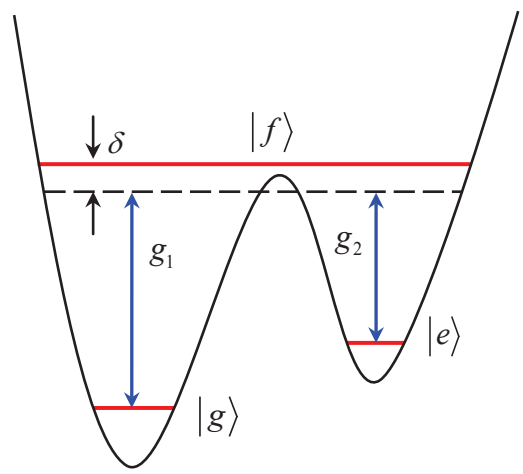

(a)

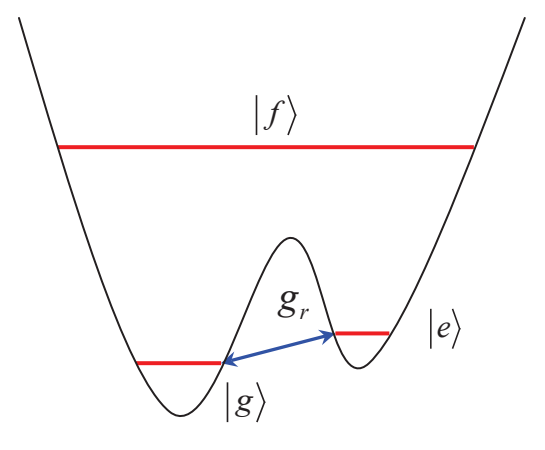

(b)

FIG. 1: (Color online) (a) Illustration of cavity 1 dispersively coupled to the $|g\rangle \leftrightarrow|f\rangle$ transition and cavity 2 dispersively coupled to the $|e\rangle \leftrightarrow|f\rangle$ transition of qutrit $A$ (a $\Lambda$-type three-level flux qutrit). The transition between the two lowest levels can be made weak by increasing the barrier between two potential wells. (b) Illustration of cavity 3 resonantly coupled to the transition between the two lowest levels of qutrit $A$. The resonant coupling constant $g_{r}$ can be increased by increasing the dipole coupling matrix element between the two lowest levels, which can be reached by lowering the potential barrier [46].

superconducting microwave resonators has been reported [41]. However, we note that how to prepare entangled states of photons in more than two cavities has not been thoroughly investigated.

In this paper, we propose a way for preparing Greenberger-Horne-Zeilinger (GHZ) entangled photon Fock states of three cavities coupled to a superconducting flux qutrit. As shown below, this proposal has the following advantages: (i) No classical microwave pulse is needed during the entanglement preparation; (ii) Neither measurement on the states of the coupler qutrit nor detection on the states of photons in cavities is required; and (iii) entangled photon Fock states of three cavities can be generated using one coupler qutrit and two steps of operation only.

To the best of our knowledge, our proposal is the first one to demonstrate that a GHZ entangled photon Fock state for three cavities can be prepared by using a superconducting flux qutrit $[24,29,42-44]$ as a coupler and without need of both classical microwave pulses and measurement. Since neither classical microwave pulse nor measurement is needed and only one coupler qutrit is used, the operation is greatly simplified, and the circuit engineering complexity and cost is much reduced, by using the present proposal. Furthermore, the method presented here is quite general, and can be applied to generate three-cavity GHZ entangled photon Fock states when the three cavities are coupled by a different three-level physical system such as a superconducting charge qutrit, a transmon qutrit, or a quantum dot.

This paper is organized as follows. In Sec. II, we introduce the Raman resonant coupling induced due to a coupler flux qutrit interacting with two cavities. In Sec. III, we discuss how to generate the GHZ entangled photon Fock state of three cavities and then give a brief discussion of the experimental issues. In Sec. IV, we give a discussion of the fidelity and possible experimental implementation. A concluding summary is given in Sec. V.

\section{RAMAN RESONANT COUPLING}

Consider a system which contains a superconducting flux qutrit $A$ and two cavities. Cavity 1 is coupled to the $|g\rangle \leftrightarrow|f\rangle$ transition with a coupling constant $g_{1}$ and a detuning $\delta=\omega_{f g}-\omega_{c 1}$ and cavity 2 is coupled to the $|e\rangle \leftrightarrow|f\rangle$ transition with a coupling constant $g_{2}$ and a detuning $\delta=\omega_{f e}-\omega_{c 2}$ [Fig. 1(a)]. Here, $\omega_{c 1}\left(\omega_{c 2}\right)$ is the frequency of cavity $1(2)$ while $\omega_{f g}\left(\omega_{f e}\right)$ is the $|g\rangle \leftrightarrow|f\rangle(|e\rangle \leftrightarrow|f\rangle)$ transition frequency of qutrit $A$. In addition, assume that each cavity is highly detuned (decoupled) from the transition between any other two levels of the qutrit $A$. In the interaction picture, the Hamiltonian of the whole system is given by

$$
H=g_{1}\left(e^{i \delta t} a_{1} S_{f g}^{+}+h . c\right)+g_{2}\left(e^{i \delta t} a_{2} S_{f e}^{+}+h . c .\right),
$$

where $S_{f g}^{+}=|f\rangle\left\langle g\left|, S_{f e}^{+}=\right| f\right\rangle\langle e|$, and $a_{1}\left(a_{2}\right)$ is the photon annihilation operator for cavity 1 (2).

Suppose that cavity 1 is dispersively coupled to the $|g\rangle \leftrightarrow|f\rangle$ transition (i.e., $\delta \gg g_{1}$ ) and cavity 2 is dispersively coupled to the $|e\rangle \leftrightarrow|f\rangle$ transition (i.e., $\delta \gg g_{2}$ ). Under this condition, the Hamiltonian (1) reduces to

$$
H_{\text {eff }}=H_{0}+H_{I}
$$


with

$$
\begin{gathered}
H_{0}=-\frac{g_{1}^{2}}{\delta} a_{1}^{+} a_{1}|g\rangle\left\langle g\left|-\frac{g_{2}^{2}}{\delta} a_{2}^{+} a_{2}\right| e\right\rangle\langle e|, \\
H_{I}=-\frac{g_{1} g_{2}}{\delta}\left(a_{1}^{+} a_{2} S_{e g}^{-}+h . c .\right),
\end{gathered}
$$

where $S_{e g}^{-}=|g\rangle\langle e|$. The two terms in Eq. (3) are ac-stark shifts of the energy levels $|g\rangle$ and $|e\rangle$ which are induced by the mode of cavity 1 and the mode of cavity 2, respectively; while Eq. (4) describes the Raman resonant $|g\rangle \leftrightarrow|e\rangle$ coupling caused due to the two-cavity cooperation.

Performing an unitary transform $U=e^{-i H_{0} t}$, we obtain

$$
\begin{aligned}
\widetilde{H}_{I} & =U^{+} H_{I} U \\
& =-\frac{g_{1} g_{2}}{\delta}\left(e^{-i \frac{g_{1}^{2}}{\delta} a_{1}^{+} a_{1} t} a_{1}^{+} a_{2} S_{e g}^{-} e^{i \frac{g_{2}^{2}}{\delta} a_{2}^{+} a_{2} t}+\text { h.c. }\right) .
\end{aligned}
$$

Denote $|0\rangle_{j}$ and $|1\rangle_{j}$ as the vacuum state and the single-photon state for cavity $j(j=1,2)$, respectively. One can see that under the Hamiltonian (5), the state $|e\rangle|0\rangle_{1}|1\rangle_{2}$ evolves within a Hilbert space formed by the two orthogonal states $|e\rangle|0\rangle_{1}|1\rangle_{2}$ and $|g\rangle|1\rangle_{1}|0\rangle_{2}$. In this Hilbert space, the Hamiltonian (5) can be expanded as

$$
\widetilde{H}_{I}=\left(\begin{array}{cc}
0 & -e^{i \varphi t} g_{1} g_{2} / \delta \\
-e^{-i \varphi t} g_{1} g_{2} / \delta & 0
\end{array}\right)
$$

where $\varphi=g_{1}^{2} / \delta-g_{2}^{2} / \delta$. For $g_{1}=g_{2}=g$ (achievable by tuning the coupling capacitance $C_{1}$ between qutrit $A$ and cavity 1 as well as the coupling capacitance $C_{2}$ between qutrit $A$ and cavity 2, see Fig. 2 below), the matrix (6) becomes

$$
\widetilde{H}_{I}=\left(\begin{array}{cc}
0 & -g^{2} / \delta \\
-g^{2} / \delta & 0
\end{array}\right)
$$

One can easily find that under the Hamiltonian (7), the time evolution of the state $|e\rangle|0\rangle_{1}|1\rangle_{2}$ is described by

$$
|e\rangle|0\rangle_{1}|1\rangle_{2} \rightarrow \cos \left(\frac{g^{2}}{\delta} t\right)|e\rangle|0\rangle_{1}|1\rangle_{2}+i \sin \left(\frac{g^{2}}{\delta} t\right)|g\rangle|1\rangle_{1}|0\rangle_{2} .
$$

We now return to the original interaction picture by applying an unitary transformation $e^{-i H_{0} t}$ to the right side of Eq. (8). It can be found that in the original interaction picture, the time evolution of the state $|e\rangle|0\rangle_{1}|1\rangle_{2}$ remains the same as Eq. (8), except for a common phase $e^{i g^{2} t / \delta}$ which appears on both terms $|e\rangle|0\rangle_{1}|1\rangle_{2}$ and $|g\rangle|1\rangle_{1}|0\rangle_{2}$ of Eq. (8).

The result (8) obtained here will be employed for the preparation of the three-cavity GHZ entangled photon Fock states, as discussed below.

\section{PREPARING THREE-CAVITY GHZ-TYPE ENTANGLED PHOTON FOCK STATES}

Consider a superconducting flux qutrit $A$ coupled to three cavities, which is illustrated in Fig. 2. Initially, qutrit $A$ is decoupled from each cavity, which can be reached by a prior adjustment of the level spacings of qutrit $A$, such that each cavity is highly detuned from the transition between any two levels of qutrit $A$. Note that for a superconducting qutrit, the level spacings can be rapidly adjusted by varying external control parameters (e.g., magnetic flux applied to phase, transmon, or flux qutrits, see e.g. Refs. [17], [44], and [45]). In addition, assume that qutrit $A$ is initially in the state $|e\rangle$; and cavities 1 and 3 are initially in the vacuum state while cavity 2 is initially in a single-photon state.

The procedure for generating the GHZ entangled photon Fock state of triple cavities is listed as follows.

Step (i): Adjust the level spacing of qutrit $A$ such that cavity 3 is decoupled from qutrit $A$ but cavity 1 is dispersively coupled to the $|g\rangle \leftrightarrow|f\rangle$ transition and cavity 2 is dispersively coupled to the $|e\rangle \leftrightarrow|f\rangle$ transition, as shown in Fig. 1(a). When the conditions mentioned in Sec. II are met, the time evolution of the state $|e\rangle|0\rangle_{1}|1\rangle_{2}$ is given by Eq. (8), from which one can see that if qutrit $A$ interacts with cavities 1 and 2 for a given time $t_{1}=\delta \pi /\left(4 g^{2}\right)$, then the initial state $|e\rangle|0\rangle_{1}|1\rangle_{2}|0\rangle_{3}$ of the whole system evolves into 


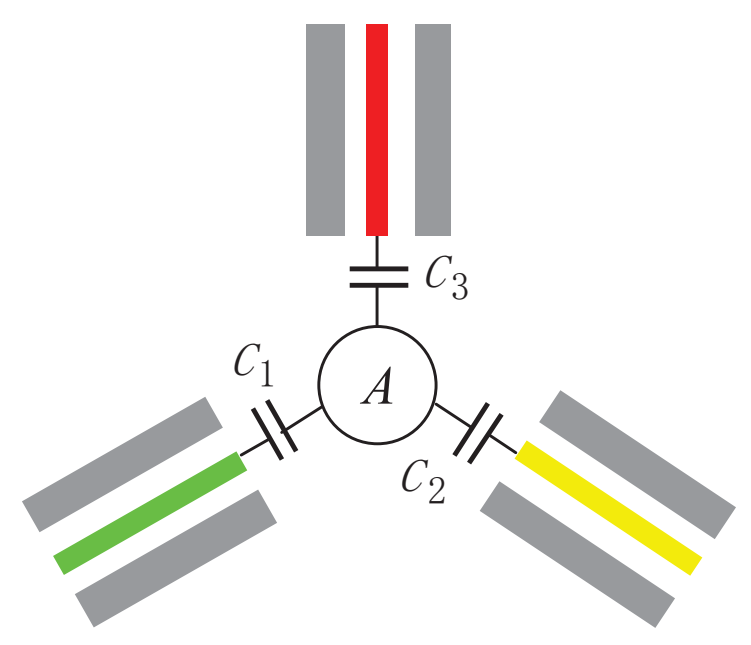

FIG. 2: (Color online) Setup for triple cavities coupled by a superconducting flux qutrit. Each cavity here is a one-dimensional coplanar waveguide transmission line resonator. The circle A represents a superconducting flux qutrit, which is capacitively coupled to cavity $j$ via a capacitance $C_{j}(j=1,2,3)$.

$$
\frac{1}{\sqrt{2}}\left(|e\rangle|0\rangle_{1}|1\rangle_{2}+i|g\rangle|1\rangle_{1}|0\rangle_{2}\right)|0\rangle_{3}
$$

Step (ii): Adjust the level spacing of qutrit $A$ such that cavities 1 and 2 are now decoupled from qutrit $A$, but cavity 3 is resonant with the transition between the two lowest levels $|g\rangle$ and $|e\rangle$ of qutrit $A$ [Fig. 1(b)], with a resonant coupling constant $g_{r}$ (which can be increased by rapidly lowering the potential barrier between the two potential wells [46]). One can see that if qutrit $A$ interacts with cavity 3 for a given time $t_{2}=\pi /\left(2 g_{r}\right)$, the state $|g\rangle|0\rangle_{3}$ remains unchanged while the state $|e\rangle|0\rangle_{3}$ changes to $-i|g\rangle|1\rangle_{3}$ in the interaction picture [47]. As a result, the state (9) becomes

$$
(-i) \frac{1}{\sqrt{2}}\left(|0\rangle_{1}|1\rangle_{2}|1\rangle_{3}-|1\rangle_{1}|0\rangle_{2}|0\rangle_{3}\right)|g\rangle .
$$

The result (10) demonstrates that a three-cavity GHZ entangled photon Fock state is prepared while qutrit $A$ is disentangled from each cavity after the above operations. Note that after the operation of step (ii), the level spacings of qutrit $A$ need to be adjusted back to the original configuration (the one before the entanglement preparation), such that qutrit $A$ is decoupled from the cavities. Thus, after the operation, the prepared GHZ state is maintained because of no qutrit-cavity interaction.

For the method to work, the following conditions need to be satisfied:

(i) The occupation probability $p$ of the level $|f\rangle$ for the qutrit during step (i) is given by $p \simeq 4 /\left[4+(\delta / g)^{2}\right][48]$, which needs to be negligibly small in order to reduce the operation error.

(ii) The total operation time $\tau$, given by

$$
\tau=\delta \pi /\left(4 g^{2}\right)+\pi /\left(2 g_{r}\right)+3 t_{d}+t_{b}
$$

(where $t_{d}$ is the typical time required for adjusting the qutrit level spacings while $t_{b}$ is the typical time required for adjusting the potential barrier), needs to be much shorter than the energy relaxation time $T_{1}$ and dephasing time $T_{2}$ of the levels $|e\rangle$ (note that the level $|f\rangle$ is almost not occupied during the operation), such that decoherence, caused due to energy relaxation and dephasing process of the qutrit, is negligible during the operation.

(iii) For cavity $i(i=1,2,3)$, the lifetime of the cavity mode is given by $T_{c a v}^{i}=\left(Q_{i} / \omega_{c i}\right) / \bar{n}_{i}$, where $Q_{i}$ and $\bar{n}_{i}$ are the (loaded) quality factor and the average photon number of cavity $i$, respectively. For the three cavities here, the lifetime of the cavity modes is given by

$$
T_{\text {cav }}=\frac{1}{3} \min \left\{T_{\text {cav }}^{1}, T_{\text {cav }}^{2}, T_{\text {cav }}^{3}\right\}
$$


which should be much longer than $\tau$, such that the effect of cavity decay is negligible during the operation.

(iii) There exists an intercavity crosstalk coupling between any two cavities, e.g., cavities $k$ and $l$, during each step of the operation, which however can be made negligible as long as the cavity-cavity frequency detuning $\Delta_{k l}$ is much larger than the intercavity crosstalk coupling constant $g_{k l}$ between cavities $k$ and $l(k l=12,13,23)$.

Before ending this section, we should point out that adjusting the level spacings of qutrit $A$ is unnecessary. Alternatively, the method can be implemented with adjusting the frequencies of the cavities. It should be noticed that rapid tuning of cavity frequencies has been demonstrated recently in superconducting microwave cavities (e.g., in less than a few nanoseconds for a superconducting transmission line resonator [49]).

\section{FIDELITY}

The proposed protocol for creating the state (10) involves two basic steps of operation as discussed above.

(i) During the operation of step (i), cavity 1 is dispersively coupled to the $|g\rangle \leftrightarrow|f\rangle$ transition and cavity 2 is dispersively coupled to the $|e\rangle \leftrightarrow|f\rangle$ transition, while cavity 3 requires to be decoupled from qutrit $A$. In the interaction picture, the interaction Hamiltonian governing this operation is given by

$$
\begin{aligned}
H_{I, 1} & =g_{1}\left(e^{i \delta t} a_{1} S_{f g}^{+}+h . c\right)+g_{2}\left(e^{i \delta t} a_{2} S_{f e}^{+}+h . c .\right) \\
& +\sum_{j=1,3} g_{j f e}\left(e^{i \Delta_{j f e} t} a_{j} S_{f e}^{+}+\text {h.c. }\right) \\
& +\sum_{j=2,3} g_{j f g}\left(e^{i \Delta_{j f g} t} a_{j} S_{f g}^{+}+h . c .\right) \\
& +\sum_{j=1,2,3} g_{j e g}\left(e^{i \Delta_{j e g} t} a_{j} S_{e g}^{+}+h . c .\right) \\
& +\sum_{k l=12,13,23} g_{k l}\left(e^{i \Delta_{k l} t} a_{k} a_{l}^{+}+h . c .\right)
\end{aligned}
$$

where the subscript $j$ represents cavity $j, S_{e g}^{+}=|e\rangle\langle g|$, and $\Delta_{k l}=\omega_{c l}-\omega_{c k}$ with $\omega_{c l}\left(\omega_{c k}\right)$ being the frequency of cavity $l(k)$. Let us give some explanation on Eq. (13). The two terms in the first line describe the dispersive interaction between cavity 1 and the $|g\rangle \leftrightarrow|f\rangle$ transition as well as the dispersive interaction between cavity 2 and the $|e\rangle \leftrightarrow|f\rangle$ transition, which induce the Raman resonant coupling between the two lowest levels $|g\rangle \leftrightarrow|e\rangle$ transition of qutrit $A$, as discussed in Sec. II. The term in the second line represents the unwanted off-resonant coupling between cavity $j(j=1,3)$ and the $|e\rangle \leftrightarrow|f\rangle$ transition, with a coupling constant $g_{j f e}$ and detuning $\Delta_{j f e}=\omega_{f e}-\omega_{c j}$. The term in the third line indicates the unwanted off-resonant coupling between cavity $j(j=2,3)$ and the $|g\rangle \leftrightarrow|f\rangle$ transition, with a coupling constant $g_{j f g}$ and detuning $\Delta_{j f g}=\omega_{f g}-\omega_{c j}$. The term in the fourth line represents the unwanted off-resonant coupling between cavity $j(j=1,2,3)$ and the $|g\rangle \leftrightarrow|e\rangle$ transition, with a coupling constant $g_{j e g}$ and detuning $\Delta_{j e g}=\omega_{e g}-\omega_{c j}$ ( $\omega_{e g}$ is the $|g\rangle \leftrightarrow|e\rangle$ transition frequency). The term in the last line represents the unwanted intercavity crosstalk between any two of three cavities.

(ii) During the operation of step (ii), both cavities 1 and 2 require to be decoupled from qutrit $A$, while cavity 3 is resonant with the $|g\rangle \leftrightarrow|e\rangle$ transition. In the interaction picture, the interaction Hamiltonian for this operation is given by

$$
\begin{aligned}
H_{I, 2}= & g_{r}\left(a_{3} S_{e g}^{+}+h . c .\right)+\sum_{j=1,2} g_{j e g}^{\prime}\left(e^{i \Delta_{j e g}^{\prime} t} a_{j} S_{e g}^{+}+\text {h.c. }\right) \\
& +\sum_{j=1,2,3} g_{j f g}^{\prime}\left(e^{i \Delta_{j f g}^{\prime} t} a_{j} S_{f g}^{+}+\text {h.c. }\right) \\
& +\sum_{j=1,2,3} g_{j f e}^{\prime}\left(e^{i \Delta_{j f e}^{\prime} t} a_{j} S_{f e}^{+}+\text {h.c. }\right)+\varepsilon
\end{aligned}
$$

where the first term in the first line represents the resonant interaction between cavity 3 and the $|g\rangle \leftrightarrow|e\rangle$ transition; all other terms are the unwanted interaction between the qutrit and cavities; and $\varepsilon$ is the last term in Eq. (13) for the unwanted intercavity crosstalk. In addition, $g_{j k l}^{\prime}$ is the coupling constant between cavity $j$ and the $|l\rangle \leftrightarrow|k\rangle$ transition of qutrit $A(k l=f g, f e, e g)$, and $\Delta_{j k l}^{\prime}=\omega_{k l}^{\prime}-\omega_{c j}$ is the detuning between the frequency $\omega_{c j}$ of cavity $j$ and the adjusted $|l\rangle \leftrightarrow|k\rangle$ transition frequency $\omega_{k l}^{\prime}$.

When the dissipation and dephasing are included, the dynamics for the $k$ th step of operation is determined by 


$$
\begin{aligned}
\frac{d \rho}{d t}= & -i\left[H_{I, k}, \rho\right]+\sum_{j=1}^{3} \kappa_{j} \mathcal{L}\left[a_{j}\right]+\gamma_{k \varphi, f e}\left(S_{f e}^{z} \rho S_{f e}^{z}-\rho\right) \\
& +\gamma_{k f e} \mathcal{L}\left[S_{f e}^{-}\right]+\gamma_{k \varphi, f g}\left(S_{f g}^{z} \rho S_{f g}^{z}-\rho\right)+\gamma_{k f g} \mathcal{L}\left[S_{f g}^{-}\right] \\
& +\gamma_{k \varphi, e g}\left(S_{\text {eg }}^{z} \rho S_{\text {eg }}^{z}-\rho\right)+\gamma_{k e g} \mathcal{L}\left[S_{\text {eg }}^{-}\right],
\end{aligned}
$$

where $\mathcal{L}[\Lambda]=\Lambda \rho \Lambda^{+}-\Lambda^{+} \Lambda \rho / 2-\rho \Lambda^{+} \Lambda / 2$, with $\Lambda=a_{j}, S_{f e}^{-}, S_{f g}^{-}, S_{e g}^{-} ; S_{f e}^{z}=|f\rangle\langle f|-| e\rangle\left\langle e\left|, S_{f g}^{z}=\right| f\right\rangle\langle f|-| g\rangle\langle g|$, and $S_{e g}^{z}=|e\rangle\langle e|-| g\rangle\langle g|$. In addition, $\kappa_{j}$ is the decay rate of the mode of cavity $j(j=1,2,3), \gamma_{k \varphi, f e}\left(\gamma_{k \varphi, f g}\right)$ and $\gamma_{k f e}$ $\left(\gamma_{k f g}\right)$ are the dephasing rate and the energy relaxation rate of the level $|f\rangle$ of qutrit $A$ for the decay path $|f\rangle \rightarrow|e\rangle$ $(|g\rangle)$, respectively and $\gamma_{k \varphi, e g}$ and $\gamma_{k e g}$ are those of the level $|e\rangle$ for the decay path $|e\rangle \rightarrow|g\rangle$ (the superscript $k$ here is used to indicate the dephasing rate and relaxation rate of qutrit $A$ during the $k$ th step of operation).

The fidelity of the operation is given by

$$
\mathcal{F}=\left\langle\psi_{i d}|\widetilde{\rho}| \psi_{i d}\right\rangle
$$

where $\left|\psi_{i d}\right\rangle$ is the state (10) of an ideal system (i.e., without dissipation, dephasing, and crosstalk) and $\widetilde{\rho}$ is the final density operator of the system when the operation is performed in a realistic physical system.

We now numerically calculate the fidelity of the prepared GHZ entangled Fock state. The parameters used in our numerical calculation for each step of operation are listed below.

Parameters for Step (i) - For a superconducting flux qutrit with three levels illustrated in Fig. 1, the typical transition frequency $\omega_{f e} / 2 \pi$ or $\omega_{f g} / 2 \pi$ is between 4 and $20 \mathrm{GHz}$, and $\omega_{e g} / 2 \pi$ can be made to be from $1 \mathrm{GHz}$ to 5 GHz. Thus, for the flux qutrit with three levels shown in Fig. 1(a) [corresponding to the operation of step (i)], we choose $\omega_{e g} / 2 \pi \sim 5 \mathrm{GHz}$ and $\omega_{f e} / 2 \pi \sim 10.0 \mathrm{GHz}$. In addition, with appropriate design of the qutrit system, one can have $\phi_{f g} \sim \phi_{f e} \sim 10 \phi_{e g}$, where $\phi_{i j}$ represents the dipole coupling matrix element between the two levels $|i\rangle$ and $|j\rangle$ with $i j \in\{f g, f e, e g\}$. As a result, we have $g_{1 e g} \sim 0.1 g_{1}, g_{1 f e} \sim g_{1} ; g_{2 e g} \sim 0.1 g_{2}, g_{2 f g} \sim g_{2} ;$ and $g_{3 e g} \sim 0.1 g_{r}$, $g_{3 f e} \sim g_{3 f g} \sim g_{r}$ [50]. Here, $g_{1}=g_{2}=g$ (set previously). Other parameters used in the numerical calculation for this step of operation are: (i) $g_{r} / 2 \pi=200 \mathrm{MHz}$, (ii) $\delta / 2 \pi=1 \mathrm{GHz}$, (iii) $\gamma_{1 \varphi, f e}^{-1}=\gamma_{1 \varphi, f g}^{-1}=\gamma_{1 \varphi, e g}^{-1}=1 \mu \mathrm{s}$, $\gamma_{1 f g}^{-1} \sim \gamma_{1 f e}^{-1}=10 \mu \mathrm{s}, \gamma_{1 e g}^{-1}=100 \mu \mathrm{s}[51,52]$, (iv) $\omega_{c 1} / 2 \pi \sim 14 \mathrm{GHz}, \omega_{c 2} / 2 \pi \sim 9.0 \mathrm{GHz}$, and $\omega_{c 3} / 2 \pi \sim 1.0 \mathrm{GHz}$, (v) $\kappa_{1}^{-1}=\kappa_{2}^{-1}=\kappa_{3}^{-1}=10 \mu \mathrm{s}$.

Parameters for Step (ii) - For the flux qutrit with three levels shown in Fig. 1(b) [associated with the operation of step (ii)], we choose $\omega_{e g} / 2 \pi \sim 1.0 \mathrm{GHz}$ and $\omega_{f e} / 2 \pi \sim 12.0 \mathrm{GHz}$. Note that for a three-level flux qutrit, lowering the potential barrier would slightly change the dipole coupling matrix element between the two levels $|g\rangle$ and $|f\rangle$ or $|e\rangle$ and $|f\rangle$ but significantly increase the dipole coupling matrix element between the two lowest levels $|g\rangle$ and $|e\rangle$. Thus, we can assume $\phi_{f g}^{\prime} \sim \phi_{f g}, \phi_{f e}^{\prime} \sim \phi_{f e}$, and $\phi_{e g}^{\prime} \sim 10 \phi_{e g}$, where $\phi_{i j}^{\prime}$ represents the dipole coupling matrix element between the two levels $|i\rangle$ and $|j\rangle$ for the level structure shown in Fig. 1(b), with $i j \in\{f g, f e, e g\}$. As a result, we have $g_{1 e g}^{\prime} \sim g_{1 f g}^{\prime} \sim g_{1 f e}^{\prime} \sim g_{1}, g_{2 e g}^{\prime} \sim g_{2 f g}^{\prime} \sim g_{2 f e}^{\prime} \sim g_{2}$, and $g_{3 f g}^{\prime} \sim g_{3 f e}^{\prime} \sim g_{r}$. Other parameters used in the numerical calculation for this step of operation are: (i) $\gamma_{2 \varphi, f e}^{-1}=\gamma_{2 \varphi, f g}^{-1}=\gamma_{2 \varphi, e g}^{-1}=1 \mu \mathrm{s}, \gamma_{2 f g}^{-1} \sim \gamma_{2 f e}^{-1} \sim \gamma_{2 e g}^{-1}=10 \mu \mathrm{s}$, and (ii) the same values of $\omega_{c 1}, \omega_{c 2}, \omega_{c 3}, \kappa_{1}^{-1}, \kappa_{2}^{-1}, \kappa_{3}^{-1}$ as those used for the operation of step (i).

For the parameters chosen here, the fidelity versus $b \equiv \delta / g$ is shown in Fig. 3 , for $g_{k l}=0,0.4 g_{r}, 0.6 g_{r}, 0.8 g_{r}, g_{r}$. Fig. 3 shows that for $b \leq 12$, when $g_{k l} \leq 0.4 g_{r}$, the effect of intercavity cross coupling between cavities on the fidelity of the prepared GHZ states is negligible, which can be seen by comparing the top two lines. In addition, one can see from Fig. 3 that for $b \sim 8$, a high fidelity $\sim 98.5 \%$ can be achieved when $g_{k l} \leq 0.4 g_{r}$.

For $b \sim 8$, we have $g /(2 \pi) \sim 125 \mathrm{MHz}$. In addition, we set $g_{r} / 2 \pi=200 \mathrm{MHz}$ above. Note that a coupling constant $\sim 220 \mathrm{MHz}$ can be reached for a superconducting qubit coupled to a one-dimensional CPW (coplanar waveguide) resonator [13]. For the cavity frequencies chosen above and for the $\kappa_{1}^{-1}, \kappa_{2}^{-1}$ and $\kappa_{3}^{-1}$ used in the numerical calculation, the required quality factors for the three cavities are $Q_{1} \sim 8.8 \times 10^{5}, Q_{2} \sim 5.7 \times 10^{5}$, and $Q_{3} \sim 6.3 \times 10^{4}$, respectively. Note that superconducting CPW resonators with a loaded quality factor $Q \sim 10^{6}$ have been experimentally demonstrated [53,54], and planar superconducting resonators with internal quality factors $Q>10^{6}$ have also been reported recently [55]. Our analysis given here demonstrates that preparation of the threecavity GHZ entangled photon Fock state is feasible within the present circuit QED technique.

This condition, $g_{k l} \leq 0.4 g_{r}$, is not difficult to satisfy with typical capacitive cavity-qutrit coupling illustrated in Fig. 2. As long as the cavities are physically well separated, the intercavity cross-talk coupling strength is $g_{k l} \sim$ $g_{r}\left(C_{k}+C_{l}\right) / C_{\Sigma}$ (for $b \sim 8, g$ is on the same order as $g_{r}$ ), where $C_{\Sigma}=\sum_{j=1}^{3} C_{j}+C_{q}$ (see Fig. 2). For $C_{j} \sim 1 \mathrm{fF}$ and $C_{\Sigma} \sim 10^{2} \mathrm{fF}$ (the typical values of the cavity-qutrit coupling capacitance and the sum of all coupling capacitance and qutrit self-capacitance, respectively), we have $g_{k l} \approx 0.02 g_{r}$. Therefore, the condition $g_{k l} \leq 0.4 g_{r}$ can be readily met in 


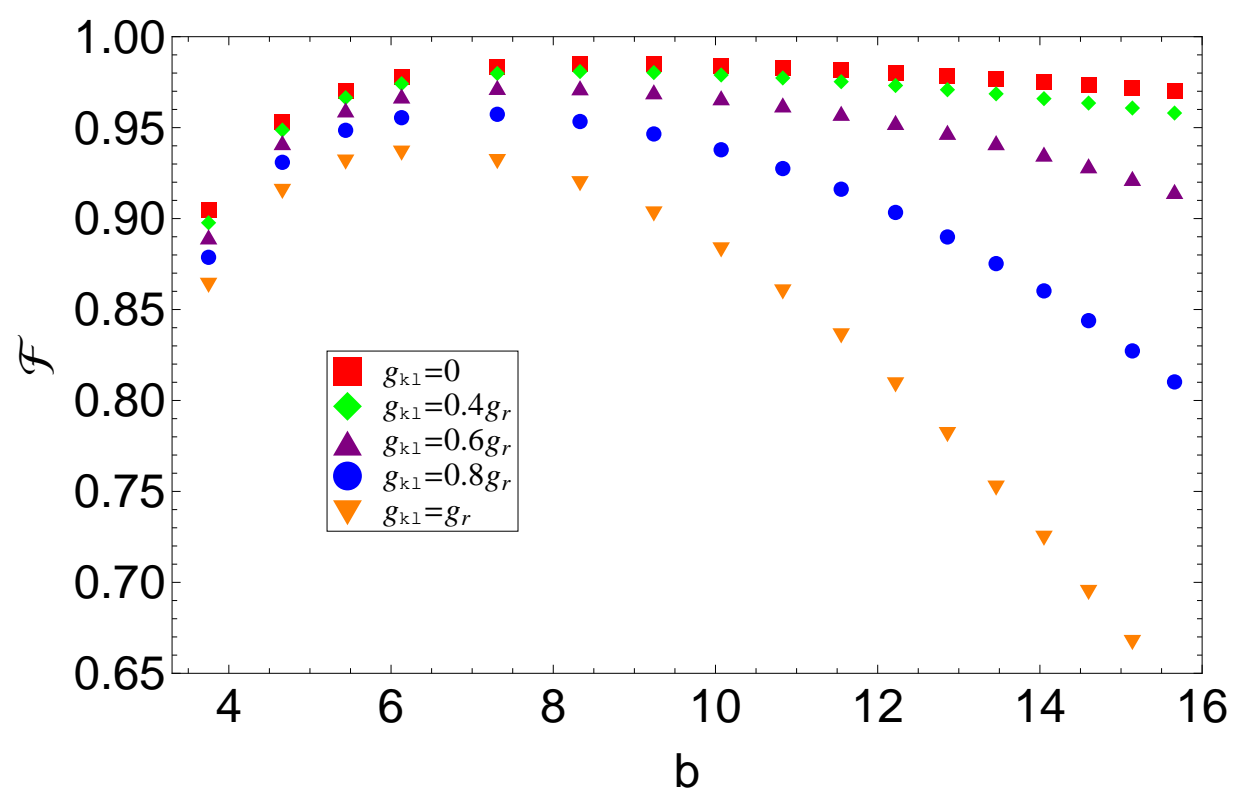

FIG. 3: (Color online) Fidelity versus $b$. Here, $b=\delta / g$. Refer to the text for the parameters used in the numerical calculation. Here, $g_{k l}$ are the coupling strengths between cavities $k$ and $l(k l=12,13,23)$, which are taken to be the same for simplicity.

experiment. Hence, implementing designs with sufficiently weak direct intercavity couplings is straightforward. We remark that further investigation is needed for each particular experimental setup. However, this requires a rather lengthy and complex analysis, which is beyond the scope of this theoretical work.

\section{CONCLUSION}

We have proposed a way for creating a three-cavity GHZ entangled photon Fock state, by using a superconducting flux qutrit coupled to the cavities. This proposal does not require the use of classical microwave pulses and any measurement during the entanglement preparation. Our numerical simulation shows that preparation of the threecavity GHZ entangled photon Fock state is feasible within the present circuit QED technique. We hope that this work could stimulate further experimental and theoretical activities. Finally, the method presented here is quite general, and can be applied to generate the same type of entangled photon Fock state for triple cavities, which are coupled by a different physical system with three levels, such as a superconducting charge qutrit, a transmon qutrit, or a quantum dot.

Before we conclude, we should mention the previous work [56] which is relevant to ours. Ref. [56] presents a scheme for the preparation of a GHZ-type entangled photon coherent state of multiple cavities by having an atom interact with each of the cavities dispersively and then measuring the state of the atom. We are aware that a three-cavity GHZ entangled photon Fock state can, in principle, be generated using the same procedure described in Ref. [56]. However, the method in Ref. [56] requires a measurement on the state of the atom; and since the prepared GHZ state depends on the measurement outcome on the atomic states, the GHZ-state preparation is not deterministic. In contrast, our present proposal mitigates these problems effectively: there is no need to measure the state of the artificial atom, and the generation of the GHZ state is deterministic. We believe that this work is of interest because no measurement is needed and also no classical microwave pulse is required, which greatly simplifies the operation and reduces the engineering complexity and cost.

\section{ACKNOWLEDGMENTS}

We thank Yang Yu for many fruitful discussions. This work was supported in part by the National Natural Science Foundation of China under Grant No. 11074062, the Zhejiang Natural Science Foundation under Grant No. LZ13A040002, the Open Fund from the SKLPS of ECNU, and the funds from Hangzhou Normal University under Grant No. HSQK0081. Q.P. Su was supported by Zhejiang Provincial Natural Science Foundation of China (Grant No. LQ12A05004). Z. F. Zheng was supported by Student Scientific Research Foundation of Hangzhou Normal 
University under Grant No. 1283XXM101.

[1] Y. F. Huang, B. H. Liu, L. Peng, Y. H. Li, L. Li, C. F. Li and G. C. Guo: Nat. Commun. 2 (2011) 546; X. C. Yao, T. X. Wang, P. Xu, H. Lu, G. S. Pan, X. H. Bao, C. Z. Peng, C. Y. Lu, Y. A. Chen, and J. W. Pan: Nat. Photonics 6 (2012) 225 .

[2] T. Monz, P. Schindler, J. T. Barreiro, M. Chwalla, D. Nigg, W. A. Coish, M. Harlander, W. Hansel, M. Hennrich, and R. Blatt: Phys. Rev. Lett. 106 (2011) 130506.

[3] D. M. Greenberger, M. A. Horne, and A. Zeilinger: in Bells Theorem, Quantum Theory and Conceptions of the Universe, edited by M. Kafatos (Kluwer Academic, Dordrecht, 1989).

[4] E. Hagley, X. Maitre, G. Nogues, C. Wunderlich, M. Brune, J. M. Raimond, and S. Haroche: Phys. Rev. Lett. 79 (1997) 1; S. Osnaghi, P.Bertet, A. Auffeves, P. Maioli, J. M. Raimond, and S. Haroche: Phys. Rev. Lett. 87 (2001) 037902.

[5] A. Rauschenbeutel, G. Nogues, S. Osnaghi, P. Bertet, M. Brune, J. M. Raimond, and S. Haroche: Science 288 (2000) 2024.

[6] G. Chen, N. H. Bonadeo, D. G. Steel, D. Gammon, D. S. Katzer, D. Park, and L. J. Sham: Science 289 (2000) 1906.

[7] C. P. Yang, S. I. Chu, and S. Han: Phys. Rev. A 67 (2003) 042311; C. P. Yang, S. I. Chu, and S. Han: Phys. Rev. Lett. 92 (2004) 117902; C. P. Yang, S. I. Chu, and S. Han: J. Phys.: Condens. Matter 16 (2004) 1907; C. P. Yang and S. Han: Phys. Rev. A 70 (2004) 062323; K. H. Song, Z. W. Zhou, and Guang-Can Guo: Phys. Rev. A 71 (2005) 052310; Z. Kis and E. Paspalakis: Phys. Rev. B 69 (2004) 024510; S. L. Zhu, Z. D. Wang, and P. Zanardi: Phys. Rev. Lett 94 (2005) 100502; X. L. Zhang, K. L. Gao, and M. Feng: Phys. Rev. A 74 (2006) 024303; Z. H. Peng, Y. X. Liu, Y. Nakamura, and J. S. Tsai: Phys. Rev. B 85 (2012) 024537.

[8] S. Matsuo, S. Ashhab, T. Fujii, F. Nori, K. Nagai, and N. Hatakenaka: J. Phys. Soc. Jpn 76 (2007) 054802; L. F. Wei, Y. X. Liu, M. J. Storcz, and F. Nori: Phys. Rev. A 73 (2006) 052307; L. F. Wei, Y. X. Liu, and F. Nori: Phys. Rev. Lett. 96 (2006) 246803.

[9] P. J. Leek, S. Filipp, P. Maurer, M. Baur, R. Bianchetti, J. M. Fink, M. Göppl, L. Steffen, and A. Wallraff: Phys. Rev. B 79 (2009) 180511(R).

[10] L. DiCarlo, J. M. Chow, J. M. Gambetta, L. S. Bishop, B. R. johnson, D. I. Schuster, J. Majer, A. Blais, L. Frunzio, S. M. Girvin, and R. J. Schoelkopf: Nature (London) 460 (2009) 240.

[11] M. Ansmann, H. Wang, R. C. Bialczak, M. Hofheinz, E. Lucero, M. Neeley, A. D. O'Connell, D. Sank, M. Weides, J. Wenner, A. N. Cleland, and J. M. Martinis: Nature (London) 461 (2009) 504.

[12] J. M. Chow, L. DiCarlo, J. M. Gambetta, A. Nunnenkamp, L. S. Bishop, L. Frunzio, M. H. Devoret, S. M. Girvin, and R. J. Schoelkopf: Phys. Rev. A 81 (2010) 062325.

[13] L. DiCarlo, M. D. Reed, L. Sun, B. R. Johnson, J. M. Chow, J. M. Gambetta, L. Frunzio, S. M. Girvin, M. H. Devoret, and R. J. Schoelkopf: Nature (London) 467 (2010) 574.

[14] J. Q. You and F. Nori: Physics Today 58 (11) (2005) 42; J. Q. You and F. Nori: Nature (London) 474 (2011) 589.

[15] Z. L. Xiang, S. Ashhab, J. Q. You, and F. Nori: Rev. Mod. Phys. 85 (2013) 623.

[16] I. Buluta, S. Ashhab, and F. Nori: Reports on Progress in Physics 74 (2011) 104401; S. N. Shevchenko, S. Ashhab, and F. Nori: Phys. Reports 492 (2010) 1; P.D. Nation, J.R. Johansson, M.P. Blencowe, and F. Nori: Rev. Mod. Phys. 84 (2012) 1.

[17] J. Clarke and F. K.Wilhelm: Nature (London) 453 (2008) 1031.

[18] J. Bylander, S. Gustavsson, F. Yan, F. Yoshihara, K. Harrabi, G. Fitch, D. G. Cory, Y. Nakamura, J.S. Tsai, and W. D. Oliver: Nat. Phys. 7 (2011) 565; H. Paik, D. I. Schuster, L. S. Bishop, G. Kirchmair, G. Catelani, A. P. Sears, B. R. Johnson, M. J. Reagor, L. Frunzio, L. I. Glazman, S. M. Girvin, M. H. Devoret, and R. J. Schoelkopf: Phys. Rev. Lett. 107 (2011) 240501; J. M. Chow, J. M. Gambetta, A. D. Crcoles, S. T. Merkel, J. A. Smolin, C. Rigetti, S. Poletto, George A. Keefe, M. B. Rothwell, J. R. Rozen, M. B. Ketchen, and M. Steffen: Phys. Rev. Lett. 109 (2012) 060501; C. Rigetti, J. M. Gambetta, S. Poletto, B. L. T. Plourde, J. M. Chow, A. D. Crcoles, J. A. Smolin, S. T. Merkel, J. R. Rozen, G. A. Keefe, M. B. Rothwell, M. B. Ketchen, and M. Steffen: Phys. Rev. B 86 (2012) 100506(R); R. Barends, J. Kelly, A. Megrant, D. Sank, E. Jeffrey, Y. Chen, Y. Yin, B. Chiaro, J. Mutus, C. Neill, P. OMalley, P. Roushan, J. Wenner, T. C. White, A. N. Cleland, J. M. Martinis: arXiv:1304.2322.

[19] S. Filipp1, P. Maurer, P. J. Leek, M. Baur, R. Bianchetti, J. M. Fink1, M. Goppl, L. Steffen, J. M. Gambetta, A. Blais, and A. Wallraff: Phys. Rev. Lett 102 (2009) 200402.

[20] R. C. Bialczak, M. Ansmann, M. Hofheinz, E. Lucero, M. Neeley, A. D. OConnell, D. Sank, H. Wang, J. Wenner, M. Steffen, A. N. Cleland, and J. M. Martinis: Nature Physics 6 (2010) 409.

[21] M. Neeley, R. C. Bialczak, M. Lenander, E. Lucero, M. Mariantoni, A. D. OConnell, D. Sank, H. Wang, M. Weides, J. Wenner, Y. Yin, T. Yamamoto, A. N. Cleland, and J. M. Martinis: Nature (London) 467 (2010) 570.

[22] T. Yamamoto, M. Neeley, E. Lucero, R. C. Bialczak, J. Kelly, M. Lenander, M. Mariantoni, A. D. OConnell, D. Sank, H. Wang, M. Weides, J. Wenner, Y. Yin, A. N. Cleland, and J. M. Martinis: Phys. Rev. B 82 (2010) 184515.

[23] M. D. Reed, L. DiCarlo, B. R. Johnson, L. Sun, D. I. Schuster, L. Frunzio, and R. J. Schoelkopf: Phys. Rev. Lett. 105 (2010) 173601.

[24] C. P. Yang, S. I. Chu and S. Han: Phys. Rev. A 67 (2003) 042311.

[25] J. Q. You and F. Nori: Phys. Rev. B 68 (2003) 064509. 
[26] A. Blasis, R. S. Huang, A. Wallraff, S. M. Girvin, and R. J. Schoelkopf: Phys. Rev. A 69 (2004) 062320.

[27] M. A. Sillanpaa, J. I. Park, and R. W. Simmonds: Nature(London) 449 (2007) 438.

[28] J. Majer, J. M. Chow, J. M. Gambetta, J. Koch, B. R. Johnson, J. A. Schreier, L. Frunzio, D. I. Schuster, A. A. Houck, A. Wallraff, A. Blais, M. H. Devoret, S. M. Girvin, and R. J. Schoelkopf: Nature(London) 449 (2007) 443.

[29] C. P. Yang, S. I. Chu, and S. Han: Phys. Rev. Lett. 92 (2004) 117902.

[30] A. Blais, R. S. Huang, A. Wallraff, S. M. Girvin, and R. J. Schoelkopf: Phys. Rev. A 69 (2004) 062320.

[31] A. Wallraff, D. I. Schuster, A. Blais, L. Frunzio, R. S. Huang, J. Majer, S. Kumar, S. M. Girvin, and R. J. Schoelkopf: Nature (London) 431 (2004) 162.

[32] I. Chiorescu, P. Bertet, K. Semba, Y. Nakamura, C. J. P. M. Harmans, and J. E. Mooij: Nature (London) 431 (2004) 159.

[33] F. Marquardt and C. Bruder: Phys. Rev. B 63 (2001) 054514.

[34] Y. X. Liu, L. F.Wei, and F. Nori: Europhys. Lett. 67 (2004) 941.

[35] K. Moon and S. M. Girvin: Phys. Rev. Lett. 95 (2005) 140504.

[36] F. Marquardt: Phys. Rev. B 76 (2007) 205416; M. Mariantoni, M. J. Storcz, F. K. Wilhelm, W. D. Oliver, A. Emmert, A. Marx, R. Gross, H. Christ, and E. Solano: arXiv:cond-mat/0509737.

[37] M. Mariantoni, F. Deppe, A. Marx, R. Gross, F. K. Wilhelm, and E. Solano: Phys. Rev. B 78 (2008) 104508.

[38] F. W. Strauch, K. Jacobs, and R. W. Simmonds: Phys. Rev. Lett. 105 (2010) 050501.

[39] F. W. Strauch: arXiv:quant-ph/1208.3657.

[40] S. T. Merkel and F. K. Wilhelm: New Journal of Physics 12 (2010) 093036.

[41] H. Wang, M. Mariantoni, R. C. Bialczak, M. Lenander, E. Lucero, M. Neeley, A. D. OConnell, D. Sank, M. Weides, J. Wenner, T. Yamamoto, Y. Yin, J. Zhao, J. M. Martinis, and A. N. Cleland: Phys. Rev. Lett. 106 (2011) 060401.

[42] J. Q. You, Y. X. Liu, C.P. Sun, and F. Nori: Phys. Rev. B 75 (2007) 104516.

[43] Y. X. Liu, J. Q. You, L. F. Wei, C. P. Sun, and F. Nori: Phys. Rev. Lett. 95 (2005) 087001.

[44] S. Han, J. Lapointe, and J. E. Lukens: in Single-Electron Tunneling and Mesoscopic Devices, Springer Series in Electronics and Photonics, Vol. 31 (Springer, Berlin, 1991), pp. 219 . 222.

[45] M. Neeley, M. Ansmann, R. C. Bialczak, M. Hofheinz, N. Katz, E. Lucero, A. OConnell, H. Wang, A. N. Cleland, and J. M. Martinis: Nature Phys. 4 (2008) 523.

[46] The potential barrier between the two potential wells can be rapidly adjusted in experiment (within a few nanoseconds), by varying the device critical current (Y. Yu, private communication).

[47] C. P. Yang and S. Han: Phys. Rev. A 73 (2006) 032317.

[48] C. P. Yang, S. I. Chu, and S. Han: Phys. Rev. A 70 (2004) 044303.

[49] M. Sandberg, C. M. Wilson, F. Persson, T. Bauch, G. Johansson, V. Shumeiko, T. Duty, and P. Delsing: Appl. Phys. Lett. 92 (2008) 203501.

[50] For the operation of step (ii), we assumed $g_{3 f g}^{\prime} \sim g_{3 f e}^{\prime} \sim g_{r}$ due to $\phi_{f g}^{\prime} \sim \phi_{f e}^{\prime} \sim \phi_{e g}^{\prime}$. In addition, because of $\phi_{f g}^{\prime} \sim$ $\phi_{f g}, \phi_{f e}^{\prime} \sim \phi_{f e}$, and $\phi_{e g}^{\prime} \sim 10 \phi_{e g}$, we have $g_{3 f g}^{\prime} \sim g_{3 f g}, g_{3 f e}^{\prime} \sim g_{3 f e}$, and $g_{r} \sim 10 g_{3 e g}$. Combining these, we have $g_{3 e g} \sim 0.1 g_{r}, g_{3 f e} \sim g_{3 f g} \sim g_{r}$.

[51] T1 (energy relaxation time) and T2 (dephasing time) can be made to be a few tens of $\mu$ s for the state-of-art superconducting devices (e.g. see Ref. 18).

[52] For a flux qutrit with the three levels considered, the $|g\rangle \leftrightarrow|e\rangle$ dipole matrix element is much smaller than that of the $|g\rangle \leftrightarrow|f\rangle$ and $|e\rangle \leftrightarrow|f\rangle$ transitions. Thus, $\gamma_{e g}^{-1} \gg \gamma_{f g}^{-1}, \gamma_{f e}^{-1}$.

[53] W. Chen, D. A. Bennett, V. Patel, and J. E. Lukens: Supercond. Sci. Technol. 21 (2008) 075013.

[54] P. J. Leek, M. Baur, J. M. Fink, R. Bianchetti, L. Steffen, S. Filipp, and A. Wallraff: Phys. Rev. Lett. 104 (2010) 100504.

[55] A. Megrant, C. Neill, R. Barends, B. Chiaro, Y. Chen, L. Feigl, J. Kelly, E. Lucero, M. Mariantoni, P. J. J. OMalley, D. Sank, A. Vainsencher, J. Wenner, T. C. White, Y. Yin, J. Zhao, C. J. Palmstrom, J. M. Martinis, and A. N. Cleland: Appl. Phys. Lett. 100 (2012) 113510.

[56] C. C. Gerry: Phys. Rev. A 54 (1996) R2529. 